\title{
ON SOME OPERATIONS ON GRAPH CONTINUOUS AND GRAPH QUASI-CONTINUOUS FUNCTIONS
}

\author{
Aneta MikucKa
}

\begin{abstract}
The notion of graph continuity and graph quasi-continuity was studied by Z. Grande, K. Sakálová, Przemski and A. Mikucka. In this paper, we investigate some operations on graph continuous and graph quasi-continuous function.
\end{abstract}

Let $X$ be a topological space, $(Y, \mathcal{P})$ - a uniform one, and the uniformity in $Y$ be induced by a family of pseudometrics $\mathcal{P}$. In this paper, we denote the graph of a function $f: X \rightarrow(Y, \mathcal{P})$ by $G(f)$, and the symbol $\overline{G(f)}$ stands for the closure of $G(f)$. The letter $\mathcal{R}$ stands for the set of real numbers. A set $A \subset X$ is said to be :

- semi-open if $A \subset \overline{\operatorname{Int} A}$, [7];

- semi-closed, if $X \backslash A$ is semi-open, [2];

- semi-neighborhood of $x_{0}$ if $A$ is semi-open and $x_{0} \in A$.

LEMMA 1 ([8]).

(a) Each nowhere dense set is semi-closed.

(b) If a set $W$ is semi-open and dense, and a set $A$ is dense, then the set $W \cap A$ is dense.

Definition 1. A function $f: X \rightarrow Y$ is said to be:

- quasi-continuous at $x_{0} \in X$ if, for each $\varrho \in \mathcal{P}, \varepsilon>0$ and for each neighborhood $U$ of $x_{0}$, there exists a nonempty open set $U_{1} \subset U$ such that $\varrho\left(f(x), f\left(x_{0}\right)\right)<\varepsilon$ for each $x \in U_{1},[10]$;

- cliquish at $x_{0}$ if, for each $\varrho \in \mathcal{P}, \varepsilon>0$ and for each neighborhood $U \subset X$ of $x_{0}$, there exists a nonempty open set $U_{1} \subset U$ such that $\varrho\left(f\left(x_{1}\right), f\left(x_{2}\right)\right)<\varepsilon$ for each $x_{1}, x_{2} \in U_{1}$, [5]. A function $f$ is called quasi-continuous (cliquish) if it has this property at each point of $X$.

(c) 2015 Mathematical Institute, Slovak Academy of Sciences.

2010 Mathematics Subject Classification: 54C08, 54 C30.

Keywords: quasicontinuity, graph quasicontinuity, graph continuity. 
Definition 2. A function $f: X \rightarrow Y$ is said to be:

- graph continuous if there exists a continuous function $g: X \rightarrow Y$ such that $G(g) \subset \overline{G(f)},[6$;

- graph quasi-continuous if there exists a quasi-continuous function $g: X \rightarrow Y$ such that $G(g) \subset \overline{G(f)}$, [8;

We will use the following notations :

$$
\begin{aligned}
A(f, g, \varrho, \varepsilon) & =\{x \in X ; \varrho(f(x), g(x))<\varepsilon\} ; \\
B(f, g, \varrho, \varepsilon) & =\{x \in X ; \varrho(f(x), g(x)) \geq \varepsilon\} ; \\
K(f(x), \varrho, \varepsilon) & =\{g(x) ; \varrho(f(x), g(x))<\varepsilon\} \quad \text { for } \varrho \in \mathcal{P} \quad \text { and } \quad \varepsilon>0 .
\end{aligned}
$$

Now, we will give the characterization of the graph quasi-continuity.

THEOREM 1 ([9]). Let $X$ be a topological space, $(Y, \mathcal{P})-$ a uniform one, and $f, g: X \rightarrow Y$.

If $g$ is quasi-continuous, then the following conditions are equivalent:

(a) $G(g) \subset \overline{G(f)}$;

(b) the set $A(f, g, \varrho, \varepsilon)$ is dense in $X$ for each $\varrho \in \mathcal{P}$ and for each $\varepsilon>0$.

THEOREM 2 ([9]). Let $X$ be a topological space, $(Y, \mathcal{P})-$ a uniform one and $f, g: X \rightarrow Y$.

If $g$ is quasi-continuous, then the following conditions are equivalent:

(a) $G(g) \subset \overline{G(f)}$ and $f$ is cliquish;

(b) the set $B(f, g, \varrho, \varepsilon)$ is nowhere dense in $X$ for each $\varrho \in \mathcal{P}$ and for each $\varepsilon>0$.

Z. Grande in [6] proved that each real function from $[0,1]$ is the sum of two graph continuous functions. Taking into consideration that the graph continuity implies the graph quasi-continuity, for the graph quasi-continuity, we have the similar situations as it is shown in [8].

Now, we consider functions with values in a locally convex topological vector space.

LEMMA 2 ([1]). In each locally convex topological space $(Y, \mathcal{T})$ there exists a family seminorm $\mathcal{S}$ with the property:

(a) for all $p_{1}, p_{2} \in \mathcal{S}$ there exists $p_{3} \in \mathcal{S}$ such that $\max \left\{p_{1}, p_{2}\right\} \leq p_{3}$,

(b) for all $p_{1} \in \mathcal{S}$ there exists $p_{2} \in \mathcal{S}$ with $p_{1}(x) \leq \frac{1}{2} p_{2}(x)$,

and such that the family $\{x ; p(x) \leq 1\}$ for $p \in \mathcal{S}$ is a neighbourhood base of the null. Moreover, the topology induced by the family $\mathcal{S}$ coincides with the topology $\mathcal{T}$. 
For $p \in \mathcal{S}$, we consider the function $\varsigma_{p}: Y \times Y \rightarrow[0, \infty)$ defined by

$$
\varsigma_{p}(x, y)=p(x-y) \text { for } \quad x, y \in Y .
$$

The function $\varsigma_{p}$ is a pseudometric for all $p \in \mathcal{S}$. We will use the symbol $\mathcal{P}_{\mathcal{S}}$ to denote the family of pseudometrics $\varsigma_{p}$. Let

$$
V\left(\varsigma_{p}, \varepsilon\right)=\{(x, y) ; p(x-y)<\varepsilon\} \quad \text { for } \quad p \in \mathcal{S}, \varepsilon>0 .
$$

Then, the family

$$
\left\{V\left(\varsigma_{p}, \varepsilon\right) ; p \in \mathcal{S}, \varepsilon>0\right\}
$$

is a base of a uniform structure on $Y$. This uniform space will be denoted by $\left(Y, \mathcal{P}_{\mathcal{S}}\right)$. A topology induced by the family $\mathcal{P}_{\mathcal{S}}$ coincides with a given topology on $Y$. So, we can consider the locally convex topological vector space as a uniform space $\left(Y, \mathcal{P}_{\mathcal{S}}\right)$.

TheOREM 3. Let $X$ be a topological space, $\left(Y, \mathcal{P}_{\mathcal{S}}\right)$ - a locally convex topological vector space. If $f_{1}: X \rightarrow Y$ is graph quasi-continuous (graph continuous), $f_{2}: X \rightarrow Y$ is graph continuous, and if at least one of them is cliquish, then $f_{1}+f_{2}: X \rightarrow Y$ is graph quasi-continuous (graph continuous).

P r o of. First, assume that the function $f_{1}$ is cliquish. Let us choose any seminorm $p \in \mathcal{S}$ and $\varepsilon>0$. Let $U \subset X$ be any nonempty open set. Since $f_{1}$ is graph quasi-continuous (graph continuous), there exists a quasi-continuous (continuous) function $g_{1}$ such that $G\left(g_{1}\right) \subset \overline{G\left(f_{1}\right)}$. From Theorem 2, we have that the set $B\left(f_{1}, g_{1}, p, \varepsilon\right)$ is nowhere dense in $X$. In the consequence, by Lemma 1(a) and Theorem 1 , the set $A\left(f_{1}, g_{1}, p, \varepsilon\right)$ is semi-open dense in $X$. From assumptions about the function $f_{2}$, there exists a continuous function $g_{2}$ such that $G\left(g_{2}\right) \subset \overline{G\left(f_{2}\right)}$. Hence, by Theorem 1 , the set $A\left(f_{2}, g_{2}, p, \varepsilon\right)$ is dense in $X$. Then, applying Lemma $1(\mathrm{~b})$, we conclude that the set $A\left(f_{1}, g_{1}, p, \varepsilon\right) \cap A\left(f_{2}, g_{2}, p, \varepsilon\right)$ is dense in $X$. Moreover, $A\left(f_{1}, g_{1}, p, \varepsilon\right) \cap A\left(f_{2}, g_{2}, p, \varepsilon\right) \subset A\left(f_{1}+f_{2}, g_{1}+g_{2}, p, 2 \varepsilon\right)$. Therefore, the set $A\left(f_{1}+f_{2}, g_{1}+g_{2}, p, 2 \varepsilon\right)$ is dense in $X$. Since the function $g_{1}+g_{2}$ is quasi-continuous (continuous), by Theorem 1 , we have

$$
G\left(g_{1}+g_{2}\right) \subset \overline{G\left(f_{1}+f_{2}\right)} \text {. }
$$

It means that the function $f_{1}+f_{2}$ is graph quasi-continuous (graph continuous).

If we take $Y=\mathcal{R}$ in the last theorem, then we get results obtained by Z. Grande in [6] and by A. Mikucka in [8].

TheOREM 4. Let $X$ be a topological space, $\left(Y, \mathcal{P}_{\mathcal{S}}\right)$ - a space with F-norm. If a function $f_{1}: X \rightarrow Y$ is graph quasi-continuous (graph continuous), a function $f_{2}: X \rightarrow Y$ is graph continuous, and if at least one of them is cliquish, then $f_{1}+f_{2}: X \rightarrow Y$ is graph quasi-continuous (graph continuous). 
The last theorem is true when we assume that $Y$ is a space with $F$-norm (observe that the notion of uniformity and the $F$-norm are independent).

Now, let $X$ be a topological space, $\left(Y, \mathcal{P}_{\mathcal{S}}\right)$ - a locally convex topological vector space. For the functions $\phi: \mathcal{R} \rightarrow \mathcal{R}$ and $f: X \rightarrow Y$, we define the function $f_{*}=\phi f: \mathcal{R} \times X \rightarrow Y$ by

$$
f_{*}(\lambda, x)=\phi(\lambda) f(x) \text { for each } \lambda \in \mathcal{R}, \quad x \in X .
$$

LEMMA 3. Let $X$ be a topological space, $\left(Y, \mathcal{P}_{\mathcal{S}}\right)-$ a locally convex topological vector space. If $f: X \rightarrow Y$ is quasi-continuous (continuous) and $\phi: \mathcal{R} \rightarrow \mathcal{R}$ is continuous, then the function $f_{*}=\phi f$ is quasi-continuous (continuous).

Proof. Let us assume that the function $f$ is graph quasi-continuous. Let us choose any seminorm $p \in \mathcal{S}, \varepsilon>0, r>0$ and any point $\left(\lambda_{0}, x_{0}\right) \in \mathcal{R} \times X$. Let $U \subset X$ be any nonempty open set and $x_{0} \in U$. Then, the set

$$
\left(\lambda_{0}-r, \lambda_{0}+r\right) \times U \text { is a neighbourhood of }\left(\lambda_{0}, x_{0}\right) .
$$

First, we consider that $p\left(f\left(x_{0}\right)\right) \neq 0$. Because the function $\phi$ is continuous at $\lambda_{0}$, we can find $\delta \in(0, r)$ such that

$$
\left|\phi\left(\lambda_{0}\right)-\phi(\lambda)\right|<\frac{\varepsilon}{2 p\left(f\left(x_{0}\right)\right)} \quad \text { for each } \quad \lambda \in\left(\lambda_{0}-\delta, \lambda_{0}+\delta\right) .
$$

Therefore,

$$
|\phi(\lambda)|<\frac{\varepsilon}{2 p\left(f\left(x_{0}\right)\right)}+\left|\phi\left(\lambda_{0}\right)\right| \text { for each } \quad \lambda \in\left(\lambda_{0}-\delta, \lambda_{0}+\delta\right) .
$$

We put $M=\frac{\varepsilon}{2 p\left(f\left(x_{0}\right)\right)}+\left|\phi\left(\lambda_{0}\right)\right|$. Hence, $M>0$. Since $f$ is quasi-continuous at $x_{0}$, there exists a nonempty open set $U_{1} \subset U$ such that $p\left(f(x)-f\left(x_{0}\right)\right)<\frac{\varepsilon}{2 M}$ for each $x \in U_{1}$. Let us select a point

$$
(\lambda, x) \in\left(\lambda_{0}-\delta, \lambda_{0}+\delta\right) \times U_{1} .
$$

Hence, we have

$$
\begin{aligned}
p\left(f_{*}(\lambda, x)-f_{*}\left(\lambda_{0}, x_{0}\right)\right) & =p\left(\phi(\lambda) f(x)-\phi\left(\lambda_{0}\right) f\left(x_{0}\right)\right) \\
& \leq p\left(\phi(\lambda) f(x)-\phi(\lambda) f\left(x_{0}\right)\right) p\left(\phi(\lambda) f\left(x_{0}\right)-\phi\left(\lambda_{0}\right) f\left(x_{0}\right)\right) \\
& =|\phi(\lambda)| p\left(f(x)-f\left(x_{0}\right)\right)+\left|\phi(\lambda)-\phi\left(\lambda_{0}\right)\right| p\left(f\left(x_{0}\right)\right) \\
& <M \frac{\varepsilon}{2 M}+\frac{\varepsilon}{2 p\left(f\left(x_{0}\right)\right)} p\left(f\left(x_{0}\right)\right)=\varepsilon .
\end{aligned}
$$

Now, we focus on $p\left(f\left(x_{0}\right)\right)=0$. Since $\phi$ is continuous at $\lambda_{0} \in \mathcal{R}$, there exists $\delta \in(0, r)$ such that $\left|\phi\left(\lambda_{0}\right)-\phi(\lambda)\right|<\varepsilon$ for each $\lambda \in\left(\lambda_{0}-\delta, \lambda_{0}+\delta\right)$. Then, $|\phi(\lambda)|<\varepsilon+\left|\phi\left(\lambda_{0}\right)\right|$ for each $\lambda \in\left(\lambda_{0}-\delta, \lambda_{0}+\delta\right)$. We put $M=\varepsilon+\left|\phi\left(\lambda_{0}\right)\right|$. Then, $M>0$. As the function $f$ is quasi-continuous at $x_{0} \in U$, we can find a nonempty 
open set $U_{1} \subset U$ such that $p\left(f(x)-f\left(x_{0}\right)\right)<\frac{\varepsilon}{M}$ for each $x \in U_{1}$. We choose $(\lambda, x) \in\left(\lambda_{0}-\delta, \lambda_{0}+\delta\right) \times U_{1}$. Summarizing the previous, we have

$$
\begin{aligned}
p\left(f_{*}(\lambda, x)-f_{*}\left(\lambda_{0}, x_{0}\right)\right) & =p\left(\phi(\lambda) f(x)-\phi\left(\lambda_{0}\right) f\left(x_{0}\right)\right) \\
& \leq p\left(\phi(\lambda) f(x)-\phi(\lambda) f\left(x_{0}\right)\right)+p\left(\phi(\lambda) f\left(x_{0}\right)-\phi\left(\lambda_{0}\right) f(x)\right) \\
& =|\phi(\lambda)| p\left(f(x)-f\left(x_{0}\right)\right)+\left|\phi(\lambda)-\phi\left(\lambda_{0}\right)\right| p\left(f\left(x_{0}\right)\right) \\
& <M \frac{\varepsilon}{M}=\varepsilon .
\end{aligned}
$$

We have shown that for each seminorm $p \in \mathcal{S}, \varepsilon>0, r>0$, for any point $\left(\lambda_{0}, x_{0}\right) \in \mathcal{R} \times X$ and for any neighbourhood $\left(\lambda_{0}-r, \lambda_{0}+r\right) \times U$ of $\left(\lambda_{0}, x_{0}\right)$, there exists a nonempty open set $\left(\lambda_{0}-\delta, \lambda_{0}+\delta\right) \times U_{1} \subset\left(\lambda_{0}-r, \lambda_{0}+r\right) \times U$ such that $p\left(f_{*}(\lambda, x)-f_{*}\left(\lambda_{0}, x_{0}\right)\right)<\varepsilon$ for each $(\lambda, x) \in\left(\lambda_{0}-\delta, \lambda_{0}+\delta\right) \times U_{1}$. It means that $f_{*}$ is quasi-continuous in $\left(\lambda_{0}, x_{0}\right)$. In case when the function $f$ is continuous, using similar arguments, we can prove the continuity of $f_{*}$.

LEMMA 4. Let $X$ be a topological space, $\left(Y, \mathcal{P}_{\mathcal{S}}\right)-$ a locally convex topological vector space. For a quasi-continuous function $g: X \rightarrow Y$ and for a continuous function $\phi: \mathcal{R} \rightarrow \mathcal{R}$, the following conditions are equivalent:

(a) $G\left(g_{*}\right) \subset \overline{G\left(f_{*}\right)}$;

(b) the set $A\left(f_{*}, g_{*}, p, \varepsilon\right)$ is dense in $\mathcal{R} \times X$ for each seminorm $p \in \mathcal{S}$ and for each $\varepsilon>0$.

P roof. $(a) \Rightarrow(b)$ Let us take a seminorm $p \in S, \varepsilon>0$, a nonempty open set $(a, b) \times U \subset \mathcal{R} \times X$, and let us choose a point $\left(\lambda_{0}, x_{0}\right) \in(a, b) \times U$. According to Lemma 3 , the function $g_{*}$ is quasi-continuous at $\left(\lambda_{0}, x_{0}\right)$. So, there exists a nonempty open set $\left(a_{1}, b_{1}\right) \times U_{1} \subset(a, b) \times U$ such that $p\left(g_{*}(\lambda, x)-g_{*}\left(\lambda_{0}, x_{0}\right)\right)<\frac{\varepsilon}{3}$ for each $(\lambda, x) \in\left(a_{1}, b_{1}\right) \times U_{1}$.

Now, let us select a point $\left(\lambda_{1}, x_{1}\right) \in\left(a_{1}, b_{1}\right) \times U_{1}$. The set

$$
\left[\left(a_{1}, b_{1}\right) \times U_{1}\right] \times K\left(g_{*}\left(\lambda_{1}, x_{1}\right), p, \frac{\varepsilon}{3}\right)
$$

is a neighbourhood of $\left(\left(\lambda_{1}, x_{1}\right), g_{*}\left(\lambda_{1}, x_{1}\right)\right)$. Because $G\left(g_{*}\right) \subset \overline{G\left(f_{*}\right)}$, we have

$$
\left[\left(\left(a_{1}, b_{1}\right) \times U_{1}\right) \times K\left(g_{*}\left(\lambda_{1}, x_{1}\right), p, \frac{\varepsilon}{3}\right)\right] \cap G\left(f_{*}\right) \neq 0 .
$$

We can find

such that

$$
\left(\left(\lambda_{2}, x_{2}\right), f_{*}\left(\lambda_{2}, x_{2}\right)\right) \in G\left(f_{*}\right)
$$

$$
\left(\lambda_{2}, x_{2}\right) \in\left(a_{1}, b_{1}\right) \times U_{1} \quad \text { and } \quad f_{*}\left(\lambda_{2}, x_{2}\right) \in K\left(g_{*}\left(\lambda_{1}, x_{1}\right), p, \frac{\varepsilon}{3}\right) .
$$


Finally, we obtain

$$
\begin{aligned}
p\left(g_{*}\left(\lambda_{2}, x_{2}\right)-f_{*}\left(\lambda_{2}, x_{2}\right)\right) \leq & p\left(g_{*}\left(\lambda_{2}, x_{2}\right)-g_{*}\left(\lambda_{0}, x_{0}\right)\right) \\
& +p\left(g_{*}\left(\lambda_{0}, x_{0}\right)-g_{*}\left(\lambda_{1}, x_{1}\right)\right) \\
& +p\left(g_{*}\left(\lambda_{1}, x_{1}\right)-f_{*}\left(\lambda_{2}, x_{2}\right)\right)<\varepsilon .
\end{aligned}
$$

It implies that

$$
\left(\lambda_{2}, x_{2}\right) \in[(a, b) \times U] \cap A\left(f_{*}, g_{*}, p, \varepsilon\right),
$$

so $A\left(f_{*}, g_{*}, p, \varepsilon\right)$ is dense in $\mathcal{R} \times X$.

$(b) \Rightarrow(a)$ Let $\left(\left(\lambda_{0}, x_{0}\right), g_{*}\left(\lambda_{0}, x_{0}\right)\right) \in G\left(g_{*}\right)$ and let $\left[\left(\lambda_{0}-r, \lambda_{0}+r\right) \times U\right] \times$ $K\left(g_{*}\left(\lambda_{0}, x_{0}\right), p, \varepsilon\right)$ be a neighbourhood of $\left(\left(\lambda_{0}, x_{0}\right), g_{*}\left(\lambda_{0}, x_{0}\right)\right)$. Since $g_{*}$ is quasicontinuous at $\left(\lambda_{0}, x_{0}\right)$, there exists a nonempty open set

$$
\begin{aligned}
& (a, b) \times U_{1} \subset\left(\lambda_{0}-r, \lambda_{0}+r\right) \times U \quad \text { with } \quad p\left(g_{*}\left(\lambda_{0}, x_{0}\right)-g_{*}(\lambda, x)\right)<\frac{\varepsilon}{2} \\
& \text { for }(\lambda, x) \in(a, b) \times U_{1} \text {. }
\end{aligned}
$$

The set $A\left(f_{*}, g_{*}, p, \frac{\varepsilon}{2}\right)$ is dense, so, $\left[(a, b) \times U_{1}\right] \cap A\left(f_{*}, g_{*}, p, \frac{\varepsilon}{2}\right) \neq 0$. Then, there exists a point $\left(\lambda_{1}, x_{1}\right) \in(a, b) \times U_{1}$ such that

$$
p\left(g_{*}\left(\lambda_{1}, x_{1}\right)-f_{*}\left(\lambda_{1}, x_{1}\right)\right)<\frac{\varepsilon}{2} .
$$

Therefore, we obtain

$$
\begin{aligned}
p\left(g_{*}\left(\lambda_{0}, x_{0}\right)-f_{*}\left(\lambda_{1}, x_{1}\right)\right) \leq & p\left(g_{*}\left(\lambda_{0}, x_{0}\right)-g_{*}\left(\lambda_{1}, x_{1}\right)\right) \\
& +p\left(g_{*}\left(\lambda_{1}, x_{1}\right)-f_{*}\left(\lambda_{1}, x_{1}\right)\right)<\varepsilon .
\end{aligned}
$$

It implies that

$$
f_{*}\left(\lambda_{1}, x_{1}\right) \in K\left(g_{*}\left(\lambda_{0}, x_{0}\right), p, \varepsilon\right) .
$$

Because

$$
\left(\lambda_{1}, x_{1}\right) \in\left(\lambda_{0}-r, \lambda_{0}+r\right) \times U
$$

and

$$
\left(\left(\lambda_{1}, x_{1}\right), f_{*}\left(\lambda_{1}, x_{1}\right)\right) \in\left[\left(\lambda_{0}-r, \lambda_{0}+r\right) \times U\right] \times K\left(g_{*}\left(\lambda_{0}, x_{0}\right), p, \varepsilon\right),
$$

we have $\left[\left(\lambda_{0}-r, \lambda_{0}+r\right) \times U\right] \cap G\left(f_{*}\right) \neq 0$. This means that

$$
\left(\left(\lambda_{0}, x_{0}\right), g_{*}\left(\lambda_{0}, x_{0}\right)\right) \in \overline{G\left(f_{*}\right)} .
$$

Hence, we have $G\left(g_{*}\right) \subset \overline{G\left(f_{*}\right)}$, which finishes the proof.

THEOREM 5. Let $X$ be a topological space, $\left(Y, \mathcal{P}_{\mathcal{S}}\right)$ - a locally convex topological vector space. If a function $f: X \rightarrow Y$ is graph quasi-continuous (graph continuous), $\phi: \mathcal{R} \rightarrow \mathcal{R}$ is continuous, then $f_{*}: \mathcal{R} \times X \rightarrow Y$ is graph quasi-continuous (graph continuous). 
P r o o f. Let us assume that $f$ is graph quasi-continuous. Let us choose a seminorm $p \in \mathcal{S}$ and $\varepsilon>0$. Let $U \subset X$ be a nonempty open set. Since a function $\phi$ is continuous at $\lambda_{0} \in \mathcal{R}$, there exists $\delta>0$ such that $\left|\phi\left(\lambda_{0}\right)-\phi(\lambda)\right|<\varepsilon$ for each $\lambda \in\left(\lambda_{0}-\delta, \lambda_{0}+\delta\right)$. Let $\lambda_{1} \in\left(\lambda_{0}-\delta, \lambda_{0}+\delta\right)$. Then, $\left|\phi\left(\lambda_{1}\right)\right|<\left|\phi\left(\lambda_{0}\right)\right|+\varepsilon$. We put $M=\left|\phi\left(\lambda_{0}\right)\right|+\varepsilon$. Then, $M>0$. Since the function $f$ is graph quasicontinuous, there exists a quasi-continuous function $g: X \rightarrow Y$ such that the set $A\left(f, g, p, \frac{\varepsilon}{M}\right)$ is dense in $X$. So, $U \cap A\left(f, g, p, \frac{\varepsilon}{M}\right) \neq \emptyset$. Hence, there exists $x_{1} \in U$ such that $p\left(f\left(x_{1}\right)-g\left(x_{1}\right)\right)<\frac{\varepsilon}{M}$. In a consequence, we have

$$
p\left(\phi\left(\lambda_{1}\right) f\left(x_{1}\right)-\phi\left(\lambda_{1}\right) g\left(x_{1}\right)\right) \leq\left|\phi\left(\lambda_{1}\right)\right| \cdot p\left(f\left(x_{1}\right)-g\left(x_{1}\right)\right)<M \cdot \frac{\varepsilon}{M}=\varepsilon .
$$

It means that $\left(\lambda_{1}, x_{1}\right) \in A(\phi f, \phi g, p, \varepsilon)$. Therefore, $\left[\left(\lambda_{0}-\delta, \lambda_{0}+\delta\right) \times U\right] \cap$ $A(\phi f, \phi g, p, \varepsilon) \neq \emptyset$. In a consequence, the set $A(\phi f, \phi g, p, \varepsilon)$ is dense in $\mathcal{R} \times X$. From Lemma 3, we have that $G\left(g_{*}\right) \subset \overline{G\left(f_{*}\right)}$ and the function $f_{*}$ is graph quasi-continuous. If the function $f$ is graph continuous, then the proof is analogous.

Corollary 1. Let $X$ be a topological space, $\left(Y, \mathcal{P}_{\mathcal{S}}\right)$-locally convex topological vector space. If a function $f: X \rightarrow Y$ is graph quasi-continuous (graph continuous) and $c \in \mathcal{R}$, then $c f: \mathcal{R} \times X \rightarrow Y$ is graph quasi-continuous (graph continuous, respectively).

For a topological space $(X, \mathcal{T})$, let $\mathcal{T}_{\alpha}=\{U \backslash H ; U \in \mathcal{T}, H$ is nowhere dense $\}$, see [11].

LEMMA 5 ([9]). Let $(X, \mathcal{T})$ be a topological space, $(Y, \mathcal{P})-$ a uniform space. A function $f:(X, \mathcal{T}) \rightarrow Y$ is graph quasi-continuous (graph continuous) if and only if a function $f:\left(X, \mathcal{T}_{\alpha}\right) \rightarrow Y$ is graph quasi-continuous (graph continuous, respectively).

Definition $3([3])$. Let $(X, \mathcal{T}),(Y, \tau)$ be topological spaces. A function $f:(X, \mathcal{T}) \rightarrow(Y, \tau)$ is a semi-homeomorphism if :

(a) the function $f$ is bijective,

(b) $f(W)$ is semi-open for each semi-open set $W \subset X$,

(c) $f^{-1}(V)$ is semi-open for each semi-open set $V \subset Y$.

LEMMA 6 ([3]). Let $(X, \mathcal{T}),(Y, \tau)$ be topological spaces. If a function $f:(X, \mathcal{T}) \rightarrow$ $(Y, \tau)$ is a semi-homeomorphism, then $f:\left(X, \mathcal{T}_{\alpha}\right) \rightarrow\left(Y, \tau_{\alpha}\right)$ is a homeomorphism.

TheOREM 6. Let $(X, \mathcal{T}),(Y, \tau)$ be topological spaces, $(Z, \mathcal{P})$-a uniform space. Then:

(a) If a function $h:(X, \mathcal{T}) \rightarrow(Y, \tau)$ is a semi-homeomorphism and $f:(Y, \mathcal{T}) \rightarrow$ $(Z, \mathcal{P})$ is graph quasi-continuous, then $f \circ h:(X, \mathcal{T}) \rightarrow(Z, \mathcal{P})$ is graph quasi-continuous. 
(b) If a function $h:(X, \mathcal{T}) \rightarrow(Y, \tau)$ is a semi-homeomorphism and $f:(Y, \mathcal{T}) \rightarrow$ $(Z, \mathcal{P})$ is graph continuous, then $f \circ h:(X, \mathcal{T}) \rightarrow(Z, \mathcal{P})$ is graph continuous.

Proof. (a) Let the function $h:(X, \mathcal{T}) \rightarrow(Y, \tau)$ is a semi-homeomorphism. From Lemma 6, we have that the function $h:\left(X, \mathcal{T}_{\alpha}\right) \rightarrow\left(Y, \tau_{\alpha}\right)$ is a homeomorphism. From the assumption and from Lemma 5 , we have that the function $f:\left(Y, \tau_{\alpha}\right) \rightarrow(Z, \mathcal{P})$ is graph quasi-continuous. So, there exists a quasi-continuous function $g:\left(Y, \tau_{\alpha}\right) \rightarrow(Z, \mathcal{P})$ such that the set $A(f, g, \varrho, \varepsilon)$ is $\tau_{\alpha}$ dense in $\left(Y, \tau_{\alpha}\right)$. Let $A(f, g, \varrho, \varepsilon)=W$ and let us take any open set $U \subset X$. So, the set $h(U)$ is open in $\left(Y, \tau_{\alpha}\right)$. Then, $W \cap h(U) \neq \emptyset$. So, there exists $y \in h(U) \cap W$. Hence,

$$
h^{-1}(y) \in U \quad \text { and } \quad h^{-1}(y) \in h^{-1}(W), \quad \text { and } \quad h^{-1}(W) \cap U \neq \emptyset .
$$

It means that the set $h^{-1}(W)$ is dense in $\left(X, \tau_{\alpha}\right)$. Let $x \in h^{-1}(W)$. Then,

$$
h(x) \in W=A(f, g, \varrho, \varepsilon) \text { and } \varrho((f \circ h)(x),(g \circ h)(x))<\varepsilon .
$$

So, $x \in A(f, g, \varrho, \varepsilon)$. In this case, we have that $h^{-1}(W) \subset A(f \circ h, g \circ h, \varrho, \varepsilon)$. Because the set $h^{-1}(W)$ is dense in $\left(X, \mathcal{T}_{\alpha}\right)$, so the set $A(f \circ h, g \circ h, \varrho, \varepsilon)$ is dense in $\left(X, \tau_{\alpha}\right)$.

Now, we prove that the function $g \circ h$ is quasi-continuous. Let us take any nonempty open set $U \subset Z$. The function $g:\left(Y, \tau_{\alpha}\right) \rightarrow(Z, \mathcal{P})$ is quasi-continuous, so the set $g^{-1}(U)$ is semi-open in $\left(Y, \tau_{\alpha}\right)$. There exists any nonempty open in $\left(Y, \tau_{\alpha}\right)$ set $V$ such that $V \subset g^{-1}(U) \subset \bar{V}$. Since the function $h:\left(X, \mathcal{T}_{\alpha}\right) \rightarrow$ $\left(Y, \tau_{\alpha}\right)$ is homeomorphism, $\left.h^{-1}(\bar{V}) \subset \overline{h^{-1}(V}\right)$, in a consequence, we have

$$
h^{-1}(V) \subset(g \circ h)^{-1}(U) \subset h^{-1}(\bar{V}) \subset \overline{h^{-1}(V)} .
$$

It means that the set $(g \circ h)^{-1}(U)$ is semi-open in $\left(X, \mathcal{T}_{\alpha}\right)$. In this way, we conclude that the set $(g \circ h)^{-1}(U)$ is semi-open in $\left(X, \mathcal{T}_{\alpha}\right)$ for each open in $\left(X, \mathcal{T}_{\alpha}\right)$ set $U$. It follows that the function $g \circ h:\left(X, \mathcal{T}_{\alpha}\right) \rightarrow(Z, \mathcal{P})$ is quasi-continuous and $G(g \circ h) \subset \overline{G(f \circ h)}$. So, we have that the function $f \circ h:\left(X, \mathcal{T}_{\alpha}\right) \rightarrow(Z, \mathcal{P})$ is graph quasi-continuous. By the Lemma 5 , we conclude that $f \circ h:(X, \mathcal{T}) \rightarrow(Z, \mathcal{P})$ is graph quasi-continuous.

(b) If the function $f:(Y, \mathcal{T}) \rightarrow(Z, \mathcal{P})$ is graph continuous, the proof is analogous.

Lemma 7 ([3]). Let $(X, \mathcal{T}),(Y, \tau)$ be topological spaces. If a function $f: X \rightarrow Y$ is a homeomorphism, then $f: X \rightarrow Y$ is a semi-homeomorphism.

Corollary 2. Let $(X, \mathcal{T}),(Y, \tau)$ be topological spaces, $(Z, \mathcal{P})$ - a uniform space. Then:

(a) If a function $h:(X, \mathcal{T}) \rightarrow Y$ is a homeomorphism and $f:(Y, \mathcal{T}) \rightarrow Z$ is graph continuous, then $f \circ h:(X, \mathcal{T}) \rightarrow Z$ is graph continuous.

(b) If a function $h:(X, \mathcal{T}) \rightarrow Y$ is a homeomorphism and $f:(Y, \mathcal{T}) \rightarrow Z$ is graph quasi-continuous, then $f \circ h:(X, \mathcal{T}) \rightarrow Z$ is graph quasi-continuous. 
TheOREM 7. Let $(X, \mathcal{T})$ be a topological space, $(Y, \mathcal{P})-a$ uniform space. Let a function $f:(X, \mathcal{T}) \rightarrow Y$ be graph quasi-continuous and cliquish. Then:

(a) The function $f_{\mid M}$ is graph quasi-continuous for each dense set $M \subset X$.

(b) The function $f_{\mid U}$ is graph quasi-continuous for each open set $U \subset X$.

Proof. (a) Let us take any $\varrho \in \mathcal{P}$ and $\varepsilon>0$. Since the function $f$ is graph quasi-continuous and cliquish, according to Theorem 2, there exists a quasi-continuous function $g$ such that the set $B(f, g, \varrho, \varepsilon)$ is nowhere dense in $X$. By a Lemma $1(\mathrm{a})$, the set $A(f, g, \varrho, \varepsilon)$ is semi-open. This fact and Lemma $1(\mathrm{~b})$ give that the set $A(f, g, \varrho, \varepsilon) \cap M=B\left(f_{\mid M}, g_{\mid M}, \varrho, \varepsilon\right)$ is dense in $X$. Since $g_{\mid M}$ is quasi-continuous, in virtue of Theorem 1, the function $f_{\mid M}$ is graph quasi-continuous.

(b) Let $x_{0} \in U$. Since $f$ is graph quasi-continuous, there exists a quasi-continuous $g$ such that $G(g) \subset \overline{G(f)}$. Let us take $\left(x_{0}, g\left(x_{0}\right)\right) \subset G(g)$ and a neighbourhood $U_{1} \times V$ of $\left(x_{0}, g\left(x_{0}\right)\right)$, where $U_{1} \subset U$. Then, $\left(U_{1} \times V\right) \cap G(f) \neq \emptyset$. We have $G\left(g_{\mid U}\right) \subset \overline{G\left(f_{\mid U}\right)}$. Because the function $g_{\mid U}$ is quasi-continuous, the function $f_{\mid U}$ is graph quasi-continuous. The proof is completed.

We can prove the following theorem in a similar way:

TheOREM 8. Let $(X, \mathcal{T})$ be a topological space and let $(Y, \mathcal{P})$ - a uniform space. Let a function $f:(X, \mathcal{T}) \rightarrow Y$ be graph continuous and cliquish. Then:

(a) The function $f_{\mid M}$ is graph continuous for each dense set $M \subset X$.

(b) The function $f_{\mid U}$ is graph continuous for each open set $U \subset X$.

Now, let $X_{1}, X_{2}$ be topological spaces and let $\left(Y_{1}, \mathcal{P}_{1}\right),\left(Y_{2}, \mathcal{P}_{2}\right)$ be uniform spaces. Then,

$$
\left(Y_{1} \times Y_{2}, \mathcal{P}_{1} \times \mathcal{P}_{2}\right)
$$

is a uniform space, where $\mathcal{P}_{1} \times \mathcal{P}_{2}$ consists of seminorms $\varrho$ such that $\varrho=\varrho_{1}+\varrho_{2}$ for some $\varrho_{1} \in \mathcal{P}_{1}, \varrho_{2} \in \mathcal{P}_{2}$. Then,

$$
\varrho\left(\left(x_{1}, x_{2}\right),\left(y_{1}, y_{2}\right)\right)=\varrho_{1}\left(x_{1}, y_{1}\right)+\varrho_{2}\left(x_{2}, y_{2}\right)
$$

for each $\left(\left(x_{1}, x_{2}\right),\left(y_{1}, y_{2}\right)\right) \in \mathcal{P}_{1} \times \mathcal{P}_{2}$. For the functions $f_{1}: X_{1} \rightarrow Y_{1}$, and $f_{2}: X_{2} \rightarrow Y_{2}$, we define the function $f_{1} \times f_{2}: X_{1} \times X_{2} \rightarrow Y_{1} \times Y_{2}$ as follows:

$$
\left(f_{1} \times f_{2}\right)\left(x_{1}, x_{2}\right)=\left(f_{1}\left(x_{1}\right), f_{2}\left(x_{2}\right)\right)
$$

for each $\left(x_{1}, x_{2}\right) \in X_{1} \times X_{2}$, (see [14]).

TheOREM 9. Let $X_{1}, X_{2}$ be topological spaces and let $\left(Y_{1}, \mathcal{P}_{1}\right),\left(Y_{2}, \mathcal{P}_{2}\right)$ be uniform spaces. If the functions $f_{1}: X_{1} \rightarrow Y_{1}, f_{2}: X_{2} \rightarrow Y_{2}$ are graph quasicontinuous (graph continuous), then $f_{1} \times f_{2}$ is graph quasi-continuous (graph continuous, respectively). 
Proof. Let us take $\varrho_{1} \in \mathcal{P}_{1}, \varrho_{2} \in \mathcal{P}_{2}$ and $\varepsilon>0$. From assumptions about functions $f_{1}, f_{2}$, there exist quasi-continuous functions $g_{1}: X_{1} \rightarrow Y_{1}$ and $g_{2}: X_{2} \rightarrow Y_{2}$ such that

$$
G\left(g_{1}\right) \subset \overline{G\left(f_{1}\right)} \text { and } G\left(g_{2}\right) \subset \overline{G\left(f_{2}\right)} .
$$

In virtue of Theorem 1 , we have that the sets

$$
A\left(f_{1}, g_{1}, \varrho_{1}, \frac{\varepsilon}{2}\right) \text { and } A\left(f_{2}, g_{2}, \varrho_{2}, \frac{\varepsilon}{2}\right)
$$

are dense in $X_{1}$ and $X_{2}$, respectively. On the other hand, we have

$$
A\left(f_{2}, g_{2}, \varrho_{2}, \frac{\varepsilon}{2}\right) \times A\left(f_{1}, g_{1}, \varrho_{1}, \frac{\varepsilon}{2}\right) \subset A\left(f_{1} \times f_{2}, g_{1} \times g_{2}, \varrho_{1}, \varepsilon\right) .
$$

In the consequence, the set $A\left(f_{1} \times f_{2}, g_{1} \times g_{2}, \varrho_{1}, \varepsilon\right)$ is dense in $X_{1} \times X_{2}$. It is know that the function $g_{1} \times g_{2}$ is quasi-continuous. So, according to Theorem 1, we have that the function $f_{1} \times f_{2}$ is graph quasi-continuous.

\section{REFERENCES}

[1] ALEXIEWICZ, A.: Analiza Funkcjonalna. PWN, Warszawa, 1969.

[2] CROSSleY, S. G.-HILDEBRAND, S. K.: Semi-closure, Texas J. Sci 22 (1971), 99-112.

[3] CROssley, S. G.-HILDEBRAND, S. K.: Semi topological properties, Fund. Math. 74 (1972), 233-254.

[4] ENGELKING, R.: General Topology. PWN, Warszawa, 1984.

[5] EWERT, J.: On quasi-continuous and cliquish maps with values in uniform spaces, Bull. Polish Acad. Sci. Math. 32 (1984), 81-88.

[6] GRANDE, Z.: Sur les fonctions A-continunes, Demonstratio Math. 11 (1978), 519-526.

[7] LEVINE, N.: Semi-open sets and semi-continuity in topological spaces, Amer. Math. Monthly 70 (1963), 36-41.

[8] MIKUCKA, A.: Graph quasi-continuity, Demonstratio Math. 36 (2003), 483-494.

[9] MIKUCKA, A.: On graph quasi-continuous functions, Tatra Mt. Math. Publ. 34 (2006), 93-105.

[10] NEUBRUNN, T.: Quasi-continuity, Real Anal. Exchange 14 (1988-1989), 259-306.

[11] NJÅSTAD, O.: On some classes of nearly open set, Pacific J. Math. 15 (1965), 961-970.

[12] SAKÁLOVÁ, K.: On structural properties of graph continuous function, Tatra Mt. Math. Publ. 6 (1996), 123-126.

[13] SAKÁLOVÁ, K.: On the maximal multiplicative family for the class of graph continuous functions, Demonstratio Math. 32 (1999), 615-620.

[14] SCHUBERT, H.: Topology. MacDonald Technical and Scientific, London, 1968.

Institute of Mathematics Academia Pomeraniensis Arciszewskiego 22 d PL-76-200 Stupsk POLAND

E-mail: aneta.mikucka@wp.pl 